F. I. Fleischer, Sur les espaces normés non-archimédiens, Neder. Akad. Wetensch. vol. 16 (1954) pp. 165-168.

G. K. A. H. Gravett, Valued linear spaces, Quart. J. Math. (Oxford) vol. 24. (1955) pp. 309-315.

I. A. W. Ingleton, The Hahn-Banach theorem for non-archimedian valued fields, Proc. Cambridge Philos. Soc. vol. 48 (1952) pp. 41-45.

S. O. F. G. Schilling, The theory of valuations, Amer. Math. Soc., New York, 1950.

NorthWESTERN UNIVERSITY

\title{
ON A PROBLEM OF D. R. HUGHES
}

\section{E. G. STRAUS AND G. SZEKERES}

D. R. Hughes (Bull. Amer. Math. Soc. vol. 63 (1957) p. 209) has proposed the following problem:

Let $G$ be a group and $p$ a prime. Define $H_{p}(G)$ to be the [normal] subgroup of $G$ generated by all the elements of $G$ which do not have order $p$. Is the following conjecture true: either $H_{p}(G)=\{1\}$ or $H_{p}(G)=G$ or $\left[G: H_{p}(G)\right]=p$ ?

He remarks that the conjecture is true for $p=2$.

In this note we prove Hughes' conjecture for $p=3$.

We shall use the following notation: if $h, g_{1}, \cdots, g_{n} \in G$ and $a_{1}, \cdots, a_{n}$ are integers then

$$
h^{a_{1} g_{1}+\cdots+a_{n} g_{n}}=g_{1}^{-1} h^{a_{1}} g_{1} g_{2}^{-1} h^{a_{2}} g_{2} \cdots g_{n}^{-1} h^{a_{n}} g_{n} .
$$

Lemma 1. If $h \in H_{p}, x \notin H_{p}$ then

$$
h^{1+x+x^{2}+\cdots+x^{p-1}}=1 .
$$

Proof. Since $x^{p-1} \notin H_{p}$, all elements of $H_{p} x^{p-1}$ have order $p$. In particular

$$
\begin{aligned}
1 & =\left(h x^{p-1}\right)^{p}=h x^{p-1} \cdot h x^{p-1} \cdots h x^{p-1} \\
& =h \cdot x^{-1} h x \cdot x^{-2} h x^{2} \cdots x^{-(p-1)} h x^{p-1}=h^{1+x+\cdots+x^{p-1}} .
\end{aligned}
$$

Lemma 2. If $h \in H_{3}$ and $x H_{3} \neq y H_{3}$ then $h^{x+y}=h^{y+x}$.

Proof. By hypothesis $z=x^{-1} y \notin H_{3}$. Hence by Lemma 1

$$
1=h_{1}^{1+z+z^{2}}=h_{1}^{1+z^{2}+s} \quad h_{1} \in H_{3}
$$

Received by the editors July 5, 1957. 
or $h_{1}^{z+z^{2}}=h_{1}^{z^{2}+z}$. Setting $h_{1}=h^{x z^{2}}$ proves the lemma.

ThEOREM. If $\left[G: H_{3}\right]>3$ then $H_{3}=\{1\}$.

Proof. Since all elements of $G / H_{3}$ are of order 3, every finitely generated subgroup of $\mathrm{G} / \mathrm{H}_{3}$ is finite by Burnside's theorem. In particular, therefore, $\mathrm{G} / \mathrm{H}_{3}$ has an Abelian subgroup of order 9. Let such a subgroup be generated by $x H_{3}, y H_{3}$. We have

$$
\begin{aligned}
3= & -\left(1+x+x^{2}\right) y-y^{2}\left(1+x+x^{2}\right)+\left(1+y+y^{2}\right) \\
& +\left(1+x y+y^{2} x^{2}\right)+\left(1+x^{2} y+y^{2} x\right)=f(x, y) .
\end{aligned}
$$

But according to Lemma 2 we have therefore $h^{3}=h^{f(x, y)}$ for every $h \in H_{3}$, and by Lemma 1 this implies $h^{3}=1$. In other words $H_{3}$ contains only the identity.

University of California, Los Angeles and

University of AdELAIDE 